\title{
The impact of fencing training symmetrisation on simple reaction time
}

\author{
Monika Johne \\ Faculty of Biomedical Sciences, Józef Piłsudski University of Physical Education, Warsaw, Poland
}

\begin{abstract}
Study aim: The symmetrisation of movements can be a way to develop individual coordinative skills, and to prevent the occurrence of injuries. For this reason, in this study an attempt was made to evaluate and compare simple reaction time and movement time for épée fencers of different sports classes, and to determine the impact that three years of symmetrisation training and unilateral training have on the speed of reaction components and on dynamical asymmetry.

Material and methods: The study was conducted on 60 women épée fencers of different sports classes, and it was repeated in two groups after three years of unilateral and symmetrisation training. Simple reaction time and movement time for the dominant and the non-dominant hand were analysed using Vienna Test System.

Results: Women épée fencers of high sports class were characterised by a significantly faster reaction time than their less experienced colleagues. In tests conducted after three years of symmetrisation training, athletes from the experimental group achieved also much better results in reaction time (RT) than those from the control group training with the unilateral method. Conclusions: Long-time unilateral training of master class women épée fencers led to dynamical asymmetry, which in the future could cause injuries and have a negative impact on the development of selected motor skills. Symmetrical training conducted in the experimental group had a positive impact on reaction time indicators as well as on movement time indicators, and it prevented the occurrence of dynamical asymmetry in the tested competitors. Thus, it can be inferred that symmetrical exercises will have a positive impact on training effectiveness and on versatility of athletes.
\end{abstract}

\section{Keywords: Asymmetry - Epee - Reaction rate - Exercise}

\section{Introduction}

In many sport disciplines, reaction time is believed to be important in achieving high sports results. The quick reaction of the player to the stimulus is determined by the motor reaction ability. It is the interval that elapses from the appearance of the stimulus to the end of the muscular activity, including: reaction time and movement time [17]. Both, reaction time and straight movement time are basic measures of the rate of information processes. Reaction time (RT) is defined as an interval between an unexpected stimulus and the onset of response. Movement time (MT), on the other hand, is initiated by the onset of response and ends upon completion of a given motor task. A separate registration of these indicators is of great practical importance because it shows temporal relations between the components of information processes, for example due to the sport level or individual aptitude of athletes [7].

Many sports studies dedicated to this issue have proven the occurrence of differences in reaction time between beginners and experienced athletes [4, 20]. Inexperienced competitors take much longer to process information in the sensory phase than the masters, compensating for the prolonged central phase by a rapid execution of the movement. On the other hand, athletes with long training experience build on this experience, significantly reducing decision-making time and reaction time, by relying on anticipative factors [10]. Defence actions taken by fencers depend on how quickly they react to various attacks of the opponent [24]. Fencers are characterised by an ability to notice early cues coming from either the body posture or 'little movements' adequate for a given motor task [19]. Besides, experienced athletes are able to select the most important stimuli, which are of key significance for a given action. A fencer on the fencing strip is forced to analyse in a fraction of a second the situation and react in a flash, which is decisive for scoring a hit and winning. In fencing, simple reaction time and choice reaction time (increasing along with the number of potential reactions of the opponent) play a key role in achieving excellent sports results $[15,21]$. 
A very interesting issue, not fully investigated yet but of great importance, is the assessment of RT with respect to the upper limb lateralisation and the development of motor skills through symmetrisation. Symmetry can be an important factor in creating a high level of technical skills and motor coordination in top-class athletes. Modern training load in sport can lead to numerous injuries, and many years of training in unilateral disciplines can have negative health consequences in the form of increased muscle cross-sectional area as well as spine and joint deformity. The solution to this problem may be symmetrical training. Movement symmetrisation is defined as a process of aligning both sides of the body, retaining however the dominance of one [22]. It is a transfer that is supposed to lead to shifting movement agility from one side of the body to the other. Many authors call it bilateral transfer. It is based on creating pathways in the nervous system and generating bi-directional movement of impulses from the right brain hemisphere to the left or the other way around [11]. Movement symmetrisation enhances harmonious physical development, develops motor versatility, helps to eliminate errors, and maybe a way of perfecting sport technique. Asymmetry and symmetry issues have been studied by many scientists attempting to evaluate their effects. However, most of these scientists focused mainly on particular types of asymmetry and symmetry, and have not carried out any comprehensive evaluation and of the impact they have on selected coordination skills. Few studies are dedicated to dynamical asymmetry in sport and still fewer publications tackle the problem comprehensively or propose some modification to the current theory of how movement is shaped [22].

Therefore, this study has attempted to evaluate and compare a simple reaction time and movement time for women épée fencers of different sports classes, and to assess the impact of three-year symmetry training and unilateral training on the rate of reaction components and on dynamical asymmetry.

\section{Materials and methods}

The study was conducted on 60 women épée fencers, who were divided into four groups. Their anthropometric characteristic is presented in Table 1. Group I included 15 medallists of European Championships and World Championships, group II - 15 medallists of Championships of Poland; whereas group III (control group) and group IV (experimental group) comprised second sports class athletes, training in two fencing clubs. Fencers from the control group participated for three years in unilateral training (traditional); they did exercises loading mainly one side of the body. Members of the experimental group participated in three year training of movement symmetrisation, which consisted in exercises loading the non-dominant side of their body. The following loads were used for symmetrisation exercises: $50 \%$ for overall exercises, $30 \%$ for focused exercises, and $20 \%$ for specialised exercises. After three years, tests were repeated in the control group and in the experimental group, to assess the impact of symmetry training on RT, MT and dynamical asymmetry.

To assess simple reaction time, Vienna Test System (VTS) was used allowing to diagnose motor coordination and neuropsychological predispositions conditioning the coordination of human movement. Indicators of simple reaction were examined for the right and left hand, using a device generating a visual stimulus showing up on the monitor. The participant put a finger on the „rest button” located on the sensor, and after noticing a given stimulus lifted the finger from the „rest button” as quickly as possible and pressed the „reaction button”. All reaction times were registered, based on which reaction time (ms) and movement time (ms) were calculated.

Test results were statistically elaborated using the STATISTICA (StatSoft, Inc.2018) software package. Consistency of statistical distributions with normal distribution was checked using the Shapiro-Wilk test. To compare measurements results in the four studied groups

Table 1. Characteristics of the groups (mean $\pm \mathrm{SD}$ )

\begin{tabular}{lcccccc}
\hline \multirow{2}{*}{ Indicators } & \multirow{2}{*}{ Group I } & \multirow{2}{*}{ Group II } & \multicolumn{2}{c}{ Group III (control) } & \multicolumn{2}{c}{ Group IV (experimental) } \\
\cline { 4 - 7 } & & & Assessment 1 & Assessment 2 & Assessment 1 & Assessment 2 \\
\hline Age [years] & $21.7 \pm 3.4$ & $17.9 \pm 2.2$ & $15.9 \pm 1.7$ & $18.9 \pm 1.7$ & $15.3 \pm 1.6$ & $18.3 \pm 1.6$ \\
Body height [cm] & $175 \pm 6.4$ & $174 \pm 5.4$ & $169.1 \pm 5.1$ & $173.4 \pm 5.7$ & $170.1 \pm 5.9$ & $175.8 \pm 7.8$ \\
Body mass [kg] & $65.3 \pm 7.3$ & $61.1 \pm 6.5$ & $56.9 \pm 6.2$ & $64.5 \pm 6.7$ & $57.4 \pm 7.9$ & $65.3 \pm 6.6$ \\
Training experience [years] & $11.2 \pm 3.7$ & $7.6 \pm 2.1$ & $3.6 \pm 1.4$ & $6.6 \pm 1.4$ & $3.2 \pm 0.6$ & $6.2 \pm 0.6$ \\
Sports class & C. i IC. & I & \multicolumn{2}{c}{ II } & \multicolumn{2}{c}{ II } \\
\hline
\end{tabular}

Note: C. - champion class; IC. - international champion class. 
of athletes, and after three years of training, the variance analysis (ANOVA) was performed for repeated measurements in the experimental group and in the control group. Assumed repeated factors were the year of testing and the body side. A significance level of $\alpha=0,05$ was assumed. Spearman coefficient was used to measure the strength of correlation between countable variables.

\section{Results}

It is commonly believed that reaction time is of key significance in achieving outstanding sports results. In the tests conducted, the most experienced and titled athletes of the master class achieved the fastest reaction time and movement time. The longest simple reaction time and movement time were observed amongst the secondsports-class athletes.

In the first study, significant differences between simple reaction time in the dominant and non-dominant hand were observed only amongst the athletes of the master sports class. This demonstrates a large dynamical asymmetry in the athletes of group I. No significant difference between the movement time results obtained by the dominant and non-dominant hand was observed for athletes of different sports classes. Tests were repeated after three years of unilateral training and symmetrisation training in the control group and in the experimental group, respectively. Also, group IV athletes achieved better RT results than those from the control group for both the dominant and non-dominant hand; the difference, however, was not statistically significant. In the second study, a considerable difference was observed between movement time of the dominant and non-dominant hand. The results obtained show that training load on the non-dominant side reduces dynamical asymmetry (Table 2).

Considerable differences in RT of the dominant hand were observed between master sports class fencers and their less experienced counterparts. Substantial differences between group I and group II athletes on the one hand, and group II and group IV athletes on the other, were also noticed in RT tests of the non-dominant hand. For athletes from the control group and the experimental group, no considerable differences were found in simple reaction time for the dominant and non-dominant hand. No substantial differences in MT of the dominant and nondominant hand were noted between the examined groups (Table 3).

Table 2. Values of the reaction time and movement time indicators for the dominant and non-dominant hand in individual groups in the first and the second study (mean $\pm \mathrm{SD}$ ) and the significance $(\mathrm{p})$ for the dominant and non-dominant hand

\begin{tabular}{|c|c|c|c|c|c|c|c|c|c|c|c|c|}
\hline \multirow[b]{2}{*}{ Index } & \multicolumn{8}{|c|}{ Assessment 1} & \multicolumn{4}{|c|}{ Assessment 2} \\
\hline & Group I & $\mathrm{p}$ & Group II & $\mathrm{p}$ & $\begin{array}{l}\text { Group III } \\
\text { (control) }\end{array}$ & $\mathrm{p}$ & $\begin{array}{c}\text { Group IV } \\
\text { (experimental) }\end{array}$ & $\mathrm{p}$ & $\begin{array}{l}\text { Group III } \\
\text { (control) }\end{array}$ & $\mathrm{p}$ & $\begin{array}{c}\text { Group IV } \\
\text { (experimental) }\end{array}$ & $\mathrm{p}$ \\
\hline RT DH [ms] & $203.3 \pm 10.9$ & & $233.6 \pm 19.8$ & & $253.1 \pm 36.1$ & & $241.4 \pm 24.3$ & & $232.4 \pm 21.3$ & & $225.6 \pm 21.3$ & \\
\hline RT NH [ms] & $219.1 \pm 21.7$ & 0.03 & $227.3 \pm 20.2$ & - & $249.2 \pm 30.3$ & - & $252.8 \pm 25.6$ & - & $241.3 \pm 26.6$ & - & $232.2 \pm 19.4$ & - \\
\hline MT DH [ms] & $98.2 \pm 18.01$ & & $97.34 \pm 18.7$ & & $106.9 \pm 25.4$ & & $107.4 \pm 13.5$ & & $98.6 \pm 15.7$ & & $100.4 \pm 12.1$ & \\
\hline MT NH [ms] & $104.4 \pm 16.7$ & - & $96.12 \pm 20.1$ & - & $116.9 \pm 31.2$ & - & $117.5 \pm 17.6$ & - & $110.2 \pm 23.1$ & 0.005 & $107.5 \pm 17.7$ & - \\
\hline
\end{tabular}

Legend: RT DH - simple reaction time for the dominant hand, RT NH - simple reaction time for the non-dominant hand, MT DH - movement time for the dominant hand, MT NH - movement time for the non-dominant hand.

Table 3. Significance of differences between the reaction time and movement time indicators for the dominant and non-dominant hand between individual groups in the first study

\begin{tabular}{|c|c|c|c|c|c|c|c|c|c|c|c|c|c|c|}
\hline Index & Group I & $\mathrm{p}$ & Group II & $\mathrm{p}$ & Group III & $\mathrm{p}$ & Group IV & $\mathrm{p}$ & Group II & Group IV & $\mathrm{p}$ & Group I & $\mathrm{p}$ & Group III \\
\hline RT DH [ms] & & 0.01 & & 0.05 & & - & & 0.01 & & & 0.01 & & 0.001 & \\
\hline RT NH [ms] & & - & & 0.05 & & - & & 0.01 & & & 0.01 & & 0.01 & \\
\hline MT DH [ms] & & - & & - & & - & & - & & & - & & - & \\
\hline MT NH [ms] & & - & & - & & - & & - & & & - & & - & \\
\hline
\end{tabular}

Legend: RT DH - simple reaction time for the dominant hand, RT NH - simple reaction time for the non-dominant hand, MT DH - movement time for the dominant hand, MT NH - movement time for the non-dominant hand. 
Table 4. Significance of differences between the control group and the experimental group in reaction time indicators and movement time indicators for the dominant and non-dominant hand obtained in the first and the second study

\begin{tabular}{|c|c|c|c|c|c|c|c|c|c|}
\hline \multirow[b]{2}{*}{ Index } & \multicolumn{3}{|c|}{ Assessment 2} & \multicolumn{3}{|c|}{ Group III (control) } & \multicolumn{3}{|c|}{ Group IV (experimental) } \\
\hline & $\begin{array}{l}\text { Group III } \\
\text { (control) }\end{array}$ & $\mathrm{p}$ & $\begin{array}{c}\text { Group IV } \\
\text { (experimetal) }\end{array}$ & Assessment 1 & $\mathrm{p}$ & Assessment 2 & Assessment 1 & $\mathrm{p}$ & Assessment 2 \\
\hline RT DH [ms] & & - & & & 0.01 & & & 0.03 & \\
\hline RT NH [ms] & & - & & & 0.05 & & & 0.01 & \\
\hline MT DH [ms] & & - & & & 0.01 & & & 0.01 & \\
\hline MT NH [ms] & & - & & & 0.01 & & & 0.01 & \\
\hline
\end{tabular}

Legend: RT DH - simple reaction time for the dominant hand, RT NH - simple reaction time for the non-dominant hand, MT DH -movement time for the dominant hand, MT NH -movement time for the non-dominant hand.

The assessment of simple reaction time after three years of unilateral training and symmetrisation training was performed in two groups: the control and the experimental one. The values of measured indicators were better for group IV athletes; the differences, however, were not statistically significant, compared to group III. The results for simple reaction time and movement time obtained by fencers from the control group and the experimental group for the dominant and non-dominant hand in the second study were significantly better than in the first study (Table 4).

\section{Discussion}

The obtained results confirm earlier studies performed on épée fencers, stating that experienced competitors make decisions much faster than their less experienced colleagues. This can be an effect of their long-time specific training and experience gained at competitions [8]. Results of studies conducted by other authors, who found no significant differences in RT between athletes of different sports classes, have also been published $[6,12]$. In a study conducted by Milic et al., it is suggested that the ability of épée fencers to quickly react to stimuli increases with their training experience. The differences between beginner and experienced épée fencers were greater the greater the selection of alternative reactions to stimuli [16]. During a combat, fencer has to react to relevant visual and tactile stimuli, and ignore some of them to select those that are most appropriate to perform a certain action. Different kinds of stimuli have different importance for a successful performance of a sports combat [4]. More technical competitors base a larger number of their actions on the so-called 'weapon intuition', and tempo fencers on visual stimuli. Interestingly, in the studies conducted on women épée fencers RT results for the non-dominant hand were also the best for athletes of high sports class; this is probably an effect of many years of training and high specialisation. Despite shortest RT achieved by the athletes for both hands, a dynamical asymmetry in simple reaction time was found only in group I. In typically unilateral sports, strong dominance of the so-called active limb in various parameters, such as reaction time, is a frequent phenomenon [14].

Fencers from group II and group III achieved better results in reaction time and movement time for the nondominant hand than the dominant one. Similar results were obtained by Badau et al. in a study on athletes doing individual sports, and also by other authors $[1,3,26]$. This may probably be an effect of using symmetrisation exercises by the coaches of these athletes. Also, a greater share of movements performed with the non-dominant hand is required from athletes practicing combat sports. Cojocariu, after analysing reaction times in athletes practicing combat sports and in a group of non-training people, observed that in the group of athletes practicing combat sports differences in reaction time for both hands were small and significantly better for the non-dominant hand, compared to the non-training group. However, in the group of athletes a much shorter RT time for the non-dominant hand was achieved, which suggests that the difference may result from systematic training and thus the frequent use of the non-dominant hand in martial arts [9]. Many researchers focusing on RT and its importance in sports assert that this indicator depends mostly on individual characteristics of the nervous and muscular system [19]. It has also been proven that reaction time can improve with specific training. Such changes were observed in a study conducted by Ando et al. where the athletes achieved increasingly shorter reaction times to visual stimuli [2]. Also, in other sport disciplines changes in reaction times can be observed (shorter RT). Piliandis et al. having tested sprinters participating in three subsequent Olympic Games have found that their reaction time improved systematically over time [18]. These results have also been confirmed by tests performed on the fencers from the control group and the experimental 
group. A major improvement in reaction time and movement time for both the dominant and non-dominant hand after three years of training was observed. The RTs of athletes training by the symmetrisation method were shorter than in the control group. It can thus be inferred that symmetrisation exercises enhance training effectiveness. Confirmation of these results can be found in a study by Thom et al. demonstrating that higher symmetry is correlated with faster reaction time [23]. Having performed a large sample study, Hope et al. have concluded that more symmetric kids have a much shorter reaction time [13]. Also, in a study conducted on male fencers, better results in the dominant hand speed movement were observed after six weeks of transfer training of the non-dominant side. The authors also demonstrated that bilateral transfer may be effective in fencing training [25].

In tests performed on the athletes from the control group a significant difference in movement time between the dominant and non-dominant hand was observed after three years of unilateral training. In the experimental group, however, such an asymmetry was not observed which also suggests that symmetrisation training prevented the occurrence of dynamical asymmetry.

\section{Conclusions}

Women épée fencers of a high sports class had a significantly faster simple reaction time than their less experienced colleagues, which is a result of specific training and their experience accumulated at sports competitions.

Many years of unilateral training of master class women épée fencers led to dynamical asymmetry in simple reaction time.

Symmetrisation training in the experimental group had a positive impact on both reaction time and movement time indicators. It can thus be concluded that symmetrisation exercises favourably impact the efficiency of training and the versatility of athletes.

Conflict of interest: Authors state no conflict of interest.

\section{References}

1. Al-Hashel J.Y., Ahmed S.F., Al-Mutairi H., Hassan S., Al-Awadhi N., Al-Saraji M. (2016) Association of Cognitive Abilities and Brain Lateralization among Primary School Children in Kuwait. Neurosci. J., 2: 1-5. DOI: 10.1155/2016/6740267.

2. Ando S, Kokubu M., Kimura T., Moritani T., Araki M. (2008) Effects of acute exercise on visual reaction time. Int. J. Sports Med., 29(12): 994-998. DOI: 10.1055/s2008-1038733.
3. Badau D., Baydil B., Badau A. (2018) Differences among Three Measures of Reaction Time Based on Hand Laterality in Individual Sports. Sports, 6 (2): 1-6. DOI:10.3390/ sports6020045.

4. Balko Š., Borysiuk Z., Šimonek J. (2016) The Influence of Different Performance Level of Fencers on Simple and Choice Reaction Time. Revista Brasileira de Cineantropometria \& Desempenho Humano 16(4): 391-400. DOI: 10.5007/1980-0037.2016v18n4p391.

5. Bolkó Š., Borysiuk Z., Bolkó I., Špulák D. (2016) The influence of different performance level of fencers on muscular coordination and reaction time during the fencing lunge. Arch. Budo, 12: 49-59.

6. Borysiuk Z. (2006) Complex evaluation of fencers predisposition in three stages of sport development. Biol. Sport, 1: 41-55.

7. Borysiuk Z. (2006) Time Structure of Information Processes in Selected Fighting Sports. AWF Warszawa.

8. Borysiuk Z., Waśkiewicz Z. (2008) Information Processes, Stimulation and Perceptual Training in Fencing. J. Hum. Kinet., 16: 63-82.

9. Cojocariu A. (2011) Measurement of reaction time in Qwan Ki Do. Biol. Sport, 28(2): 139-143. DOI: $10.5604 / 947454$.

10. Czajkowski Z. (1998) Dependence of sensorimotor response time on programming and movement complexity. Sport Wyczynowy, 3-4: 27-30.

11. Guła-Kubiszewska H., Lewandowski M. (2000) Recognising bilateral transfer in a group of high-school students] [In:] Transfer in the process of physical education] Koszyc (ed.). University of Physical Education, Warsaw, 141-150.

12. Gutiérrez-Dávila M., Rojas J., Antonio R., Navarro E. (2013) Effect of uncertainty on the reaction responser in fencing. Res. Q. Exerc. Sport, 84(1): 16-23.

13. Hope D., Bates T., Penke L., Gow A.J., Starr J.M., Deary I.J. (2013) Symmetry of the face in old age reflects childhood social status. Econ. Hum. Biol., 11: 236-244. DOI: 10.1016/j.ehb.2011.06.006.

14. Johne M., Poliszczuk T., Poliszczuk D., DąbrowskaPerzyna A. (2013) Asymmetry of Complex Reaction Time in Female Épée Fencers of Different Sports Classes. Polish Journal of Sport and Tourism, 20: 25-29. DOI:10.2478/pjst-2013-0003.

15. Klapp S.T., Erwin C.I. (1976) Relation between programming time and duration of the response being programed. Journal of Experimental Psychology. Human Perception and Performance 2: 591-598. DOI:10.1037/0096-1523 2.4.591.

16. Milic M., Nedeljkovic A., Cuk I., Mudric M., GarcíaRamos A. (2020) Comparison of reaction time between beginners and experienced fencers during quasi-realistic fencing situations. Eur. J. Sport Sci., 20: 896-905. DOI: 10.1080/17461391.2019.1671498. 
17. Mynarski W., Żywicka A. (2003) Empirical model of coordination determinants of human motor skills. AWF Katowice.

18. Pilianidis T., Mantzouranis N. (2011) Start reaction time and performance time in women's sprint at the track and fi eld world championships. Int. J. Perf. Anal. Sport, 18(3): 271-275. DOI: 10.1080/24748668.2012.11868587.

19. Roca A., Williams A.M. (2016) Expertise and the interaction between different perceptualcognitive skills: implications for testing and training. Front. Psychol., 7: 1-4. DOI: $10.3389 /$ fpsyg.2016.00792.

20. Schmidt R.A., Lee T.D. (2005) Motor control and learning: A behavioral emphasis (4th editio). Champaing, IL, USA: Human Kinetics.

21. Schmidt R.A., Lee T.D. (2011) Motor control and learning (5th ed.). Champaign, IL, USA: Human Kinetics.

22. Starosta W. (2003) Motor coordination skills. Meaning, structure, conditioning, shaping. (2th editio). Institute of Sport, Warsaw.

23. Thoma R.J., Yeo R.A., Gangestad S.W., Lewine J.D., Davis J.T. (2002) Fluctuating asymmetry and the human brain.
Laterality: Asymmetries of Body, Brain, and Cognition, 7: 45-58. DOI: 10.1080/13576500143000122.

24. Wang J. (2009) Reaction-time training for elite athletes: A winning formula for champions. Int. J. Coach. Sci., 3(2): 67-78.

25. Witkowski M., Bojkowski Ł., Karpowicz K., Konieczny M., Bronikowski M., Tomczak M. (2020) Effectiveness and Durability of Transfer Training in Fencing. Int. J. Environ. Res. Public Health, 17(3): 849. DOI: 10.3390/ ijerph17030849.

26. Yamauchi M., Imanaka K., Nakayama M., Nishizawa S. (2004) Lateral Difference and Interhemispheric Transfer on Arm-Positioning Movement between Right and Left Handers. Percept. Mot. Skills, 98(3): 1199-1209. DOI: 10.2466/PMS.98.3.1199-1209.

\section{Received 03.05.2021 \\ Accepted 17.06.2021}

(C) University of Physical Education, Warsaw, Poland 